\title{
Tek Port Laparoskopik Cerrahi Aletleri Güncelleme
}

\author{
Selçuk Şahin, Volkan Tuğcu
}

Bakırköy Dr. Sadi Konuk Eğitim ve Araştırma Hastanesi, Üroloji Kliniği, İstanbul

Tek port laparoskopik cerrahi aletleri başlıca 4 kısımda gözden geçirilebilir.

\section{1) Portlar}

a) Konvansiyonel portların kullanımı: Teknik, Caddedu ve ekibi tarafından tarif edilmiştir (1). Genişletilmiş tek bir fasyal açıklıktan çok sayıda klasik port yerleştirilerek uygulanır (Şekil 1).

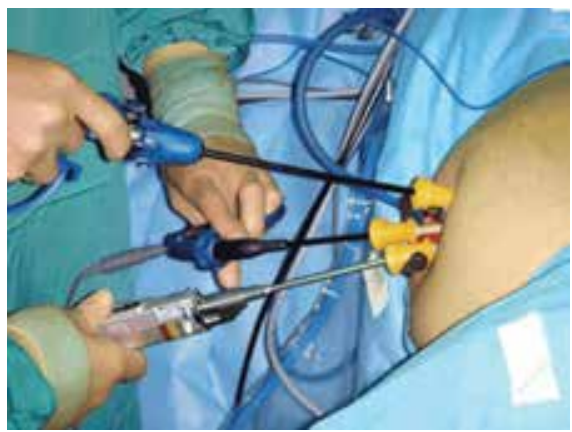

Şekil 1: Konvansiyonel portlar

Avantajları: Özel bir porta gerek olmaması, düşük maliyet, aletlerin ayrı portlardan girmesine bağlı hareket rahatlığı

Dezavantajları: Zor ve kör giriş, gerektiğinde $12 \mathrm{~mm}$ portun kullanılması zor olabilir.

b) R-Port (Olympus): İlk klinik uygulama Rane ve ark.'ları tarafından yapılmıştır (2). Cerrahın tek bir giriş yolu aracılığıyla standart laparoskopik işlemleri gerçekleştirmesini sağlayan bir cerrahi girişim sistemidir. Cihazın üzerindeki ayrı portlar karın içine aynı anda dört enstrüman girişine izin verir.

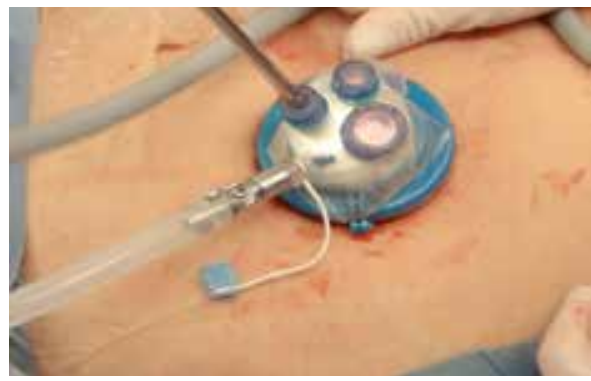

Şekil 2: R port
Avantajları: Düşük profillidir, abdomene atravmatik giriş sağlar, obez hastalarda kullanılabilir.

Dezavantajları: Tek kullanımlıktır, teleskopta kirlenme sik olur.

c) SILS Port (Covidien): Periumbilikal $1.5-2.5 \mathrm{~cm}$ insizyondan karın içine yerleştirilen üzerinde bir adet gaz girişi, iki adet $5 \mathrm{~mm}$ 'lik, bir adet $12 \mathrm{~mm}$ 'lik (gerektiğinde 15 mm'lik port ile değişebilen) port kanalları olan elastik polimer yapısında bir enstrumandır. Elastik polimer yapısı sayesinde gaz kaçakları ve operasyon esnasında porta zarar verme ihtimali en aza indirilmiştir (Şekil 3).

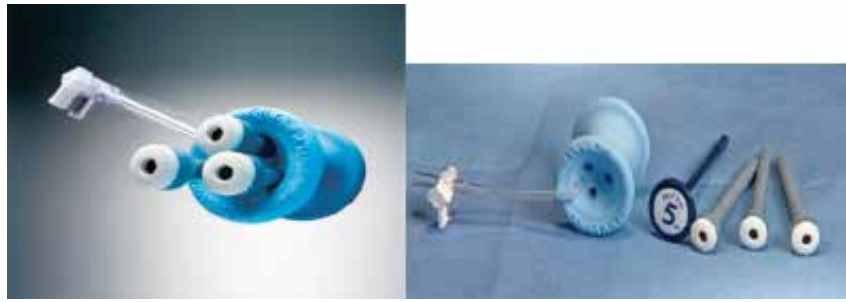

Şekil 3: SILS Port

Avantajları: Ucuzdur ve tekrar kullanılabilir, minimal gaz kaçağına neden olur.

Dezavantajları: Yerleştirmek için geniş insizyon yapılmalıdır, sabit uzunlukta olduğu için morbid obez hastalarda kullanılamaz .

d) Gel Port (Applied Medical): Retraktor ve Alexis yara koruyucudan oluşur (Şekil 4). Vücut içinde çalışma alanı dar, vücut dışında dışarıda geniştir. Trokarlar kolaylıkla yerleştirilebilir. Optik jelle direkt temas etmediğinden kirlenme azdir.

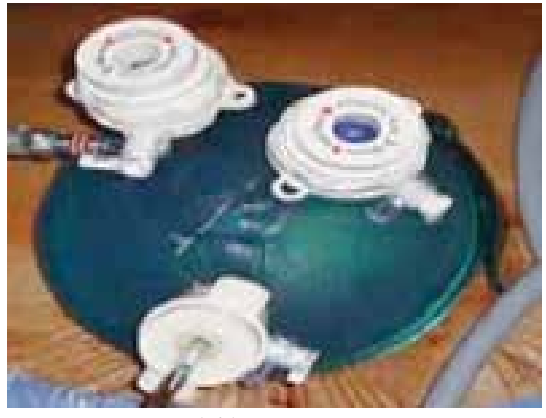

Şekil 4: Gel port 
e) Gelpoint (Applied Medical): Gelporta benzer, fakat daha küçüktür. Alet girişi jel üzerinde perforasyon yapmadan cihazın üstüne monte olan portlardan yapılır. Sütürlerin daha kolay çıkarılmasını sağlamak için bir yara koruma cihazı eklidir (Şekil 5).
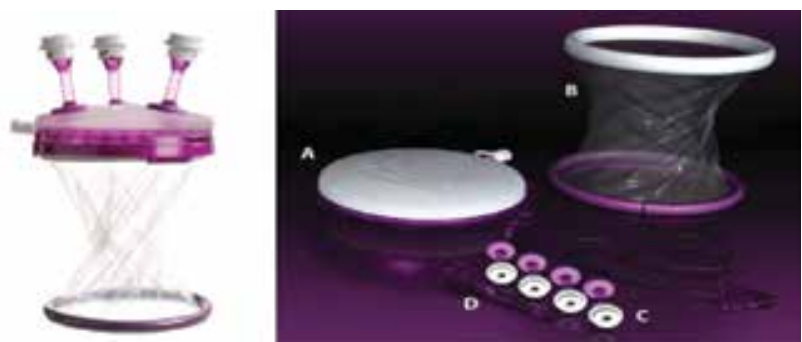

Şekil 5: Gelpoint

f) Airseal (Surgiquest): Kapağı yoktur. Bir hava perdesi oluşturarak pnömoperitonu korur. Birden fazla enstruman trokardaki büyük bir delikten kullanılabilir (Şekil 6).

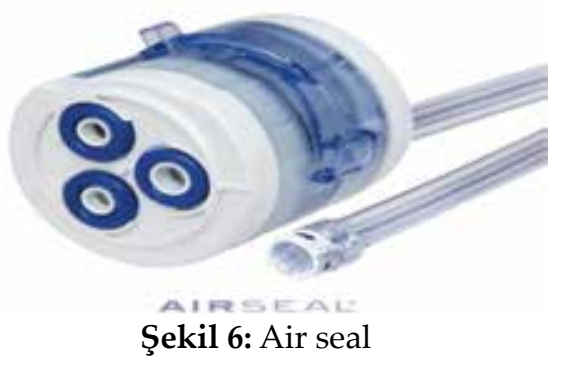

g) Single site laparoscopy access system (SSLAS, Ethicon): Üzerinde 2 adet $5 \mathrm{~mm}$ 'lik ve bir adet $12 \mathrm{~mm}$ lik (15 $\mathrm{mm}$ 'lik port opsiyonel) port giriş yeri vardır. Benzersiz $360^{\circ}$ dönen kapağı sayesinde enstruman değişimine gerek kalmadan hızlı çalışmayı sağlar (Şekil 7).

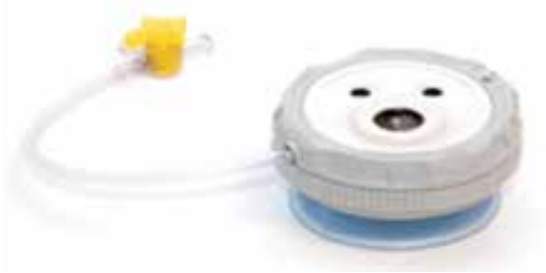

Şekil 7: SSLAS

h) S-Portal (Karl Storz) (X-Cone ve Endocone) X-Cone: Üzerinde plastik bir kapak takılı metalik konik yapıdadır. Dört enstruman portu ve bir insuflasyon portu vardır. Giriş açık Hasson tekniği ile olur. Endocone: Valvlerin
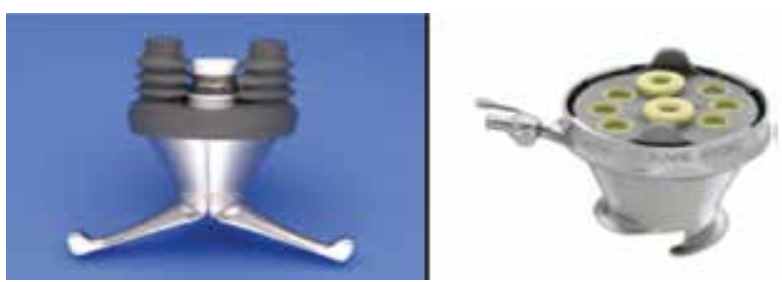

Şekil 8: X cone ve Endocone ergonomik yerleşimi birden fazla teleskop ve enstrüman erişimi için izin verir. Rijit kapağı sayesinde teleskop ve enstrumanların kontrolü kusursuzdur (Şekil 8). Her iki port da tekrar kullanilabilir.

i) Octo Port (DalimSurg): Periton içinde cilt kenarının altında bulunan alt taban plakası, yara ağzını açık tutan dış disk ve üç-dört kanallı transparan silikon koruyucudan oluşur.

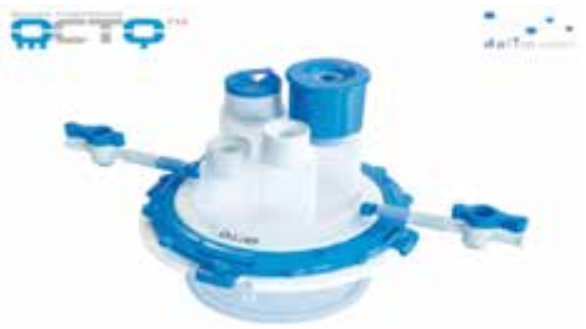

Şekil 9: Octo port

j) Ev yapımı tek port: Bir Alexis kesi retraktörünün diş halkası üzerine bir cerrahi eldiven yerleştirilir. Eldivenin 3 veya 4 parmağ kesilir ve bir adet $10 \mathrm{~mm}, 2$ veya 3 adet $5 \mathrm{~mm}$ 'lik trokar yerleştirilir. Eldivenin parmakları lastik bir bantla trokarlara bağlanır ve yara retraktörünün dış halkasına sabitlenir. Bu cihaz LESS için yeterli hareket alanı ve esneklik sağlar (3) (Şekil 10).
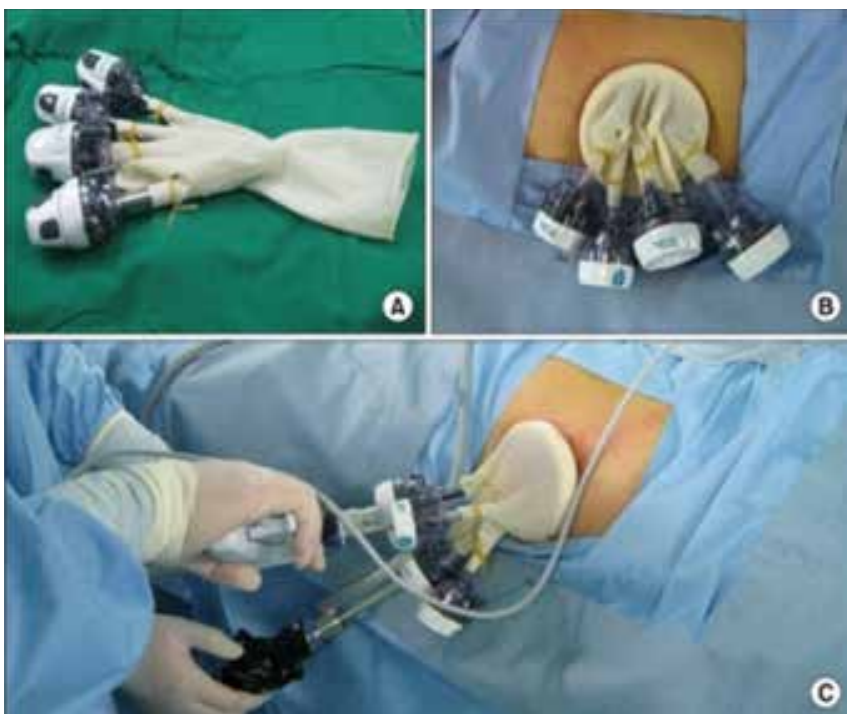

Şekil 10: Ev yapımı tek port

\section{2) Tek Port Laparoskopik Cerrahide Kullanılan Aletler}

A) Standart el aletleri: Sert yapıda ve konvansiyonel laparoskopide kullanılmak üzere geliştirilen aletlerdir (Şekil 11). Avantajları: ucuz, kullanımı kolay, kolay ulaşılabilir olmasıdır. Dezavantajları; Triangulasyon yokluğudur (5).

B) Artikulasyonlu aletler: Hareket özgürlüğ̈̈, triangulasyon sağlaması avantajı vardır. Dezavantajları ise uzun öğrenme süresi, düşük güç uygulayabilme ve maliyettir (4). 


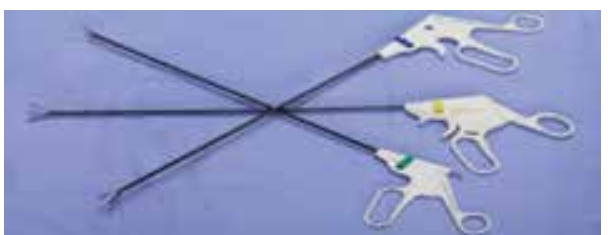

Şekil 11: Standart laparoskopi aletleri

a) RealHand, High Dexterity technology (Novare Surgical Systems Inc.): Kolu $360^{\circ}$ rotikülasyon hareketi sağlayan 5 mm'lik el aletleridir. Kullanım için öğrenme süresi oldukça uzundur (Şekil 12).

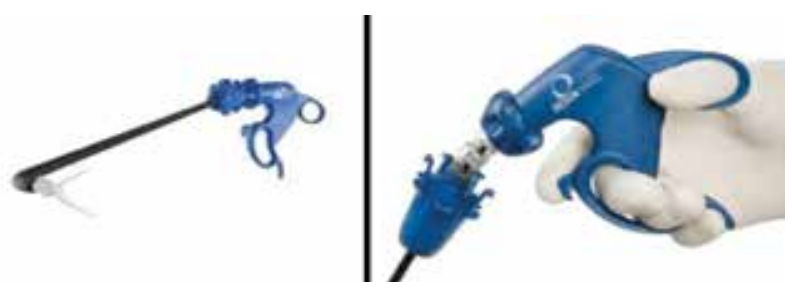

Şekil 12: Real hand

b) Autonomy, Laparo-Angle (Cambridge Endoscopic Devices Inc.): Alet $360^{\circ}$ düzlemde hareket edebilir ve istenilen pozisyonda kilitlenebilir. Büyük hacimli kolu temel dezavantajıdır (Şekil 13).

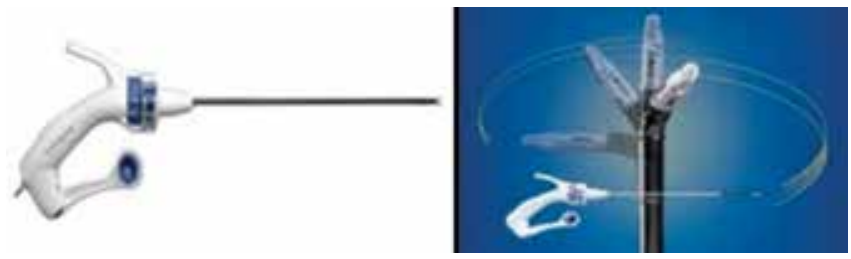

Şekil 13: Cambridge endodisektör

c) SILS Stitch (Covidien): İğne geçiş teknolojisi halihazırda klasik laparoskopide kullanılmaktadır. Ek olarak distal şaft ekleminin çene tipi rotasyon özelliği ve ek şaft uzunluğu vardır (Şekil 14).

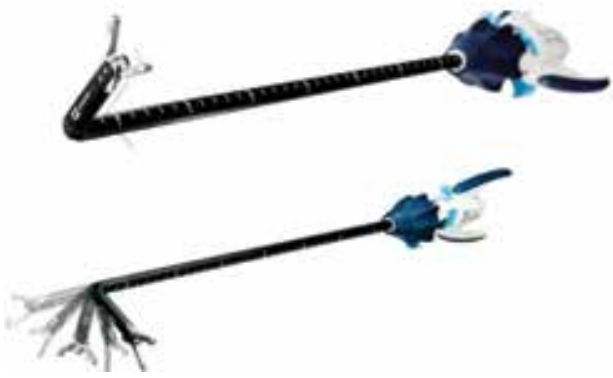

Şekil 14: SILS Stitch

d) SILS hand instruments (Covidien): Yarım küreye yakın bir alanda artikulasyon yeteneği sayesinde cerrahi alana farklı açılardan erişim sağlayabilir. İstenilen pozisyonda kilitlenebilir. Farklı şaft uzunlukları alet kollarının çarpışmasını azaltabilir.

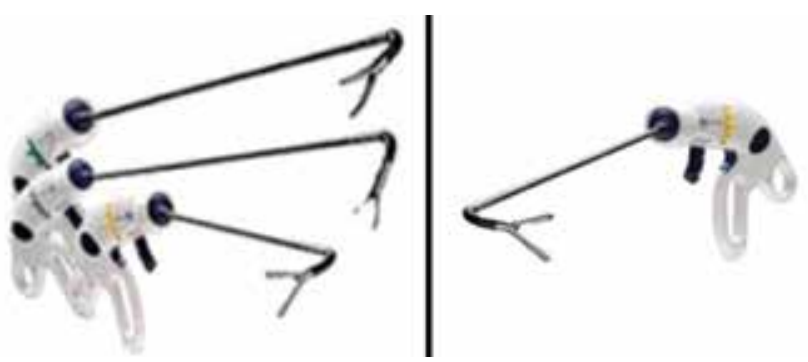

Şekil 15: SILS el aletleri

C) Bükülmüş enstrumanlar: Farklı profillerde önceden şekillendirilmiş, sert araçlardır. Tekrar kullanılabilir. Hareket özgürlüğü daha az derecedir (Şekil 16,17).

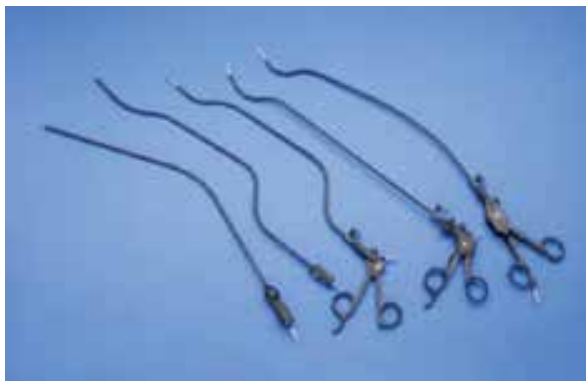

Şekil 16: S-portal serisi (Karl Storz)

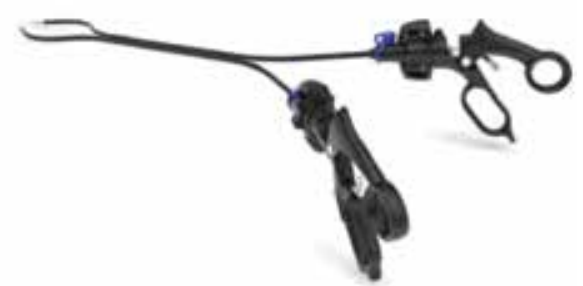

Şekil 17: HIQ LS hand instruments (Olympus)

Avantajları: Ucuz (tekrar kullanılabilir), düz aletler gibi rijiditesi vardır, triangulasyon sağlarlar.

Dezavantajları: Uzun öğrenme süresi ve özel giriş trokarları gereklidir. Traksiyon gücü sınırlıdır ve tüm bu sebepler operasyon süresini uzatır.

\section{3) Görüntüleme Sistemleri}

EndoEYE LS (Olympus): Yüksek çözünürlüklü, 5-mm, $30^{\circ}$ dijital optiktir. Doksan dereceye kadar bükülebilir. Arka ucuna ışık ve kameraya entegredir.

EndoEYE LTE VP (Olympus): Yüksek çözünürlüklü, 5-10 mm, $0^{\circ}$ dijital optiktir. $100^{\circ}$ açlanma yapabilir. Arka ucundan 1şık ve kameraya entegredir.

Ideal Eyes (Stryker): Çapı 10 mm'dir ve 1şık kablosuna entegedir. Tüm yönlere fleksiyon yapabilir.

Endo CAMeleon (Karl Storz): Ucu içinde dönen bir çip olan ve görüş kamerası $10 \mathrm{~mm}$ laparoskop ile değişken yönlerde hareket edebilen bir optiktir. ( $0^{\circ}$ ve $120^{\circ}$ arasında) 


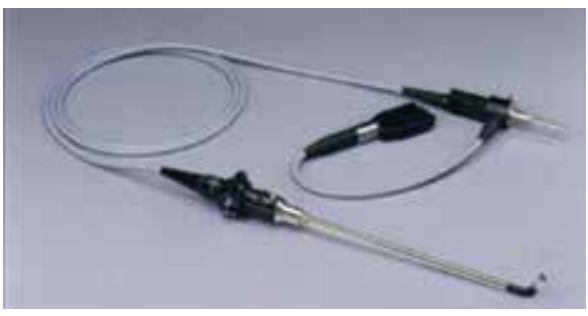

Şekil 18: Fleksibl teleskop

\section{4) Robotik Tek Port Cerrahi}

Robotik cerrahinin üç boyutlu optikleri ve tremorun azaltılması için sistemleri olmasına karşın; mevcut robotik platformlar tek port cerrahi için hantal kalmaktaydı. Fakat yeni kullanıma giren enstrumanlar ve Da Vinci Xİ modelinin piyasaya sürülmesiyle robotik tek port cerrahide gelişmeler olacağı düşünülmektedir (6) (Şekil 19).
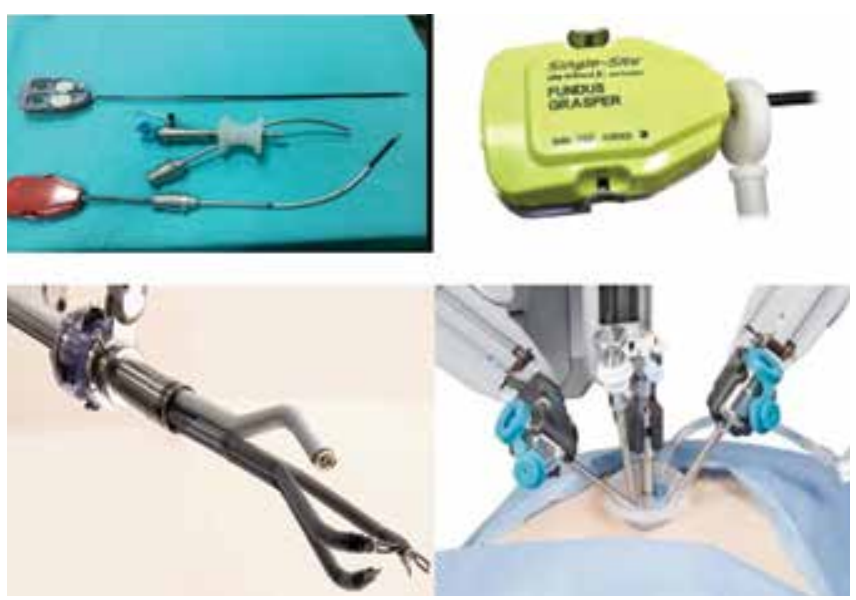

Şekil 19: Tek port robotik cerrahi enstrumanları

\section{Kaynaklar}

1. Raman JD, Cadeddu JA, Rao P, Rane A. Single-incision laparoscopic surgery: initial urological experience and comparison with natural-orifice transluminal endoscopic surgery. BJU Int 2008; 101:1493-1496.

2. Rané A, Rao P. Single-port-access nephrectomy and other laparoscopic urologic procedures using a novel laparoscopic port (R-port). Urology 2008; 72: 260-263.

3. Jiang C, Huang J, Lin T et al. Extraperitoneal transumbilical laparoendoscopic single-site radical prostatectomy using a homemade single-port device: 20 cases with midterm outcomes. World J Urol. 2014; 32:829-836.

4. Nagabhushana M, Kamath AJ, Manohar CS. Laparoendoscopic single-site surgery in urology using conventional instruments: our initial experience. J Endourol. 2013; 27:1354-60.

5. Jung JW, Cha WH, Lee BK et al. Laparoendoscopic singlesite surgery using innovative articulating instruments: preclinical evaluation of the prototype. J Endourol. 2014 28:281-285.

6. Kaouk JH, Haber GP, Autorino R et al. A Novel Robotic System for Single-port Urologic Surgery: First Clinical Investigation. Eur Urol. 2014 Jul 17 (Epub ahead of print)

Yazışma Adresi

Volkan Tu $\breve{g} c u$

Bakırköy Dr. Sadi Konuk Eğitim ve Araştırma Hastanesi, Üroloji Kliniği, İstanbul

Tel: +90 5325510729

e-mail:vtugcu@gmail.com 